\title{
Éditorial
}

\section{Montréal dans son espace}

Quelque 300 agglomérations urbaines de plus d'un million d'habitants composent l'espace planétaire, dont une vingtaine possèdent une population de plus de 10 millions. En réalité, ces agglomérations représentent les moteurs de l'économie mondiale, alimentés par diverses forces économiques, sociales, culturelles et institutionnelles. À degrés divers, ces dites « Global City-Regions » ou "régions urbaines planétaires » sont toutes en expansion rapide actuellement. Elles rayonnent plus ou moins en conséquence dans leur périphérie réciproque, souvent de dimension considérable.

Ainsi, nous pouvons considérer certaines conditions appropriées pour le positionnement mondial de Montréal et de sa région qui, certes, possèdent plusieurs atouts dans ce nouveau jeu de la globalisation des échanges. On sait que les créneaux de l'aérospatial, de l'aluminium, des technologies informationnelles, $\mathrm{du}$ pharmaceutique, des biotechnologies, du papier, avec leurs entreprises phares, excellent dans la région urbaine québécoise d'envergure planétaire dans laquelle les infrastructures de transport et de communication, les centres de R\&D, les services supérieurs spécialisés, l'enseignement supérieur, la qualité de vie jouent un rôle crucial. La masse critique de travailleurs qualifiés sur laquelle misent pertinemment les interventions des gouvernements supérieurs s'inscrit comme l'un des principaux facteurs gagnants. Sont aussi offerts des allègements fiscaux bien ciblés dans des créneaux spécifiques. Le bouillonnement culturel et l'identité québécoise représentent, en outre, une force de distinction dans le contexte de la concurrence globale.

Cette culture est par ailleurs à la base de la cohésion sociale triplement nécessaire, d'abord pour consolider la métropole québécoise elle-même, ensuite pour tisser des interfaces fertiles avec des partenaires de l'économie mondiale, et finalement pour œuvrer à l'intégration vertueuse de la Cité dans sa vaste région québécoise de rayonnement et de prélèvements de ressources. Ces trois dimensions de la cohésion désirée nécessitent des institutions appropriées au succès du positionnement de la région urbaine planétaire sise au Québec.

\section{Périphérisation de Montréal}

La localisation de Montréal et de sa région sur l'espace continental nord-américain n'est pas neutre du tout. Déjà périphérique, cette région urbaine planétaire s'avère relativement éloignée de l'actuelle principale zone mondiale de croissance ancrée sur les différents pôles asiatiques en forte émergence. En conséquence, Montréal n'a certes pas l'avantage positionnel de Vancouver ou autre Seatle.

Cette singulière "périphérisation » de Montréal et du Québec dans l'économie mondiale s'avère double en réalité. Car nous assistons, en Amérique du nord, à un lent déplacement de la population et des activités à partir des pôles jadis très prospères du nord-est (New York, Boston, Détroit...) vers les pôles fortement émergents du centre-sud des États-Unis (Albuquerque, Phoenix, Juarez, Denver...). Il s'agit de la traditionnelle mouvance vers l'ouest qui s'amplifie depuis quelques décennies en étant tirée vers le sud par les pôles forts du nord du Mexique. Ainsi s'affirme progressivement sur ce continent colonisé initialement en périphérie la centralité mue par la gravité spatiale. Centralité déjà stabilisée dans la vieille Europe avec la dorsale Londres-Turin qui contient la mégalopole européenne.

Ces puissantes forces gravitaires à l'œuvre aux échelles continentales et mondiales génèrent inévitablement d'importants effets de drainage dans les périphéries éloignées, notamment au Québec. À ce propos, l'axe du fleuve Saint-Laurent a subi un double phéno- 
mène de « renversement spatial ». D’abord historique avec l'industrialisation au milieu du $\mathrm{XIX}^{\mathrm{e}}$ siècle, Montréal a alors largement déclassé la croissance urbaine de la ville de Québec. Ensuite, un siècle plus tard, Toronto a pris le relais comme principal pôle urbain, notamment en devenant 1'hôte de nombreux services supérieurs spécialisés, dont plusieurs segments de la finance. Montréal a ainsi perdu la majeure partie de sa périphérie ouest au profit de cette nouvelle métropole canadienne, bien sûr, mais aussi pour le bénéfice de nouveaux pôles en explosion tels que Calgary, Edmonton, Vancouver. Sans compter le pôle Ottawa-Gatineau qui cherche à se positionner favorablement sur l'échiquier nord-américain. Alors que la périphérie orientale de Montréal subit des difficultés économiques majeures qui justifient leur désir de protection contre les fuites vers Montréal et Toronto, en misant stratégiquement sur des efforts de polarisation à Moncton. Du coup, le rayonnement de Montréal dans ces zones devient plus limité.

Bref, tout en étant affaiblie dans ses fonctions métropolitaines supérieures, Montréal subit davantage de compétition spatiale depuis qu'elle est devenue un pôle secondaire. Dans ce nouveau contexte contemporain, des alliances peuvent être envisagées a priori, notamment avec des pôles primaires et secondaires, limitrophes ou éloignés, dans un esprit de complémentarités dans l'interdépendance. Montréal doit aussi à l'évidence miser sur sa périphérie demeurée fidèle, soit les divers territoires qui composent le vaste espace Québec. Cette périphérie québécoise fournit des intrants (hydroélectricité, bois, capital humain...) tout en offrant un important marché aux fabricants et distributeurs de biens et services montréalais. Signalons à cet égard que la majorité des activités en opération dans la périphérie possèdent leur siège social à Montréal, y drainant ainsi de la demande et aussi des profits.

\section{La périphérie de Montréal}

On sait par ailleurs que les forces économiques très actuelles charpentent différemment la périphérie de la principale région urbaine du Québec. Existent d'abord trois phénomènes bien connus, soit l'affirmation de couronnes périurbaines dynamiques autour des villes, la dévitalisation en conséquence des zones cen- trales ainsi que le déclin relativement radical de très nombreux petits centres périphériques mono industriels. On sait par ailleurs que certaines villes se repositionnent fortement dans le système urbain modélisé jadis, notamment Drummondville, Saint-Georges, Victoriaville, Sept-Îles, Rivière-du-Loup. Et que dire des capitales régionales comme Trois-Rivières, Sherbrooke, Saguenay, Rouyn et Rimouski qui deviennent d'importants "pôles de compétitivité » en misant sur l'enseignement supérieur, la recherche, la formation professionnelle, les services spécialisés, l'incubation d'entreprises.

L'espace Québec illustre de plus de nouvelles formes territoriales dont les plus visibles sont les corridors économiques des Laurentides et de la Beauce ainsi que les technoparcs de Gatineau, de Saint-Hyacinthe, de la Vallée de la Mauricie et de la Vallée de l'aluminium au Saguenay-Lac-Saint-Jean. Les complexes récréotouristiques tels que Petite-Rivière, Mingan, Percé, Bas-Saguenay, Magog, Mont-Tremblant, etc. s'inscrivent aussi telles de nouvelles zones à saveur économique. En outre, les bassins de ressources naturelles sont, à travers leurs nécessaires rééquilibres actuels, encore truffés de nombreux petits pôles de développement qui jouent un rôle considérable dans la création de richesse collective. Notons à cet effet que, grâce à des conditions avantageuses, les grandes industries " énergivores 》 génèrent en retour le tiers du PIB québécois. Finalement, se dessine progressivement sur l'axe des Appalaches, dans le sud-est du Québec entre Saint-Jérôme et Montmagny, un véritable « croissant manufacturier » longeant la frontière américaine et fort bien positionné pour collecter des intrants et desservir le marché québécois.

Toutes ces nouvelles zones non métropolitaines jouent un rôle considérable pour l'économie du Québec et de sa métropole. Par conséquent, la cohésion globale nécessaire doit, à notre avis, reposer sur un concept général d'organisation de l'espace Québec, capable d'intégrer les diverses pièces à optimiser les potentiels dans un esprit de repositionnement mondial ancré sur Montréal et sa périphérie.

Marc-Vrbain Proulx

Université du Québec à Chicoutimi 